\title{
N-Jason: Run-Time Norm Compliance in $\operatorname{AgentSpeak}(\mathrm{L})$
}

\author{
JeeHang Lee ${ }^{1}$, Julian Padget ${ }^{1}$ \\ Brian Logan ${ }^{2}$, Daniela Dybalova ${ }^{2}$, and Natasha Alechina ${ }^{2}$ \\ ${ }^{1}$ Department of Computer Science, \\ University of Bath, \\ Bath, BA2 7AY, UK, \\ \{j. lee, j.a.padget\}@bath.ac.uk \\ 2 School of Computer Science, \\ University of Nottingham, \\ Nottingham, NG8 1BB, UK, \\ $\{\mathrm{bsl}, \mathrm{dxd}$,nza\}@cs.nott.ac.uk
}

\begin{abstract}
Normative systems offer a means to govern agent behaviour in dynamic open environments. Under the governance, agents themselves must be able to reason about compliance with state- or event-based norms (or both) depending upon the formalism used. This paper describes how norm awareness enables a BDI agent to exhibit norm compliant behaviour at run-time taking into account normative factors. To this end, we propose $\mathbf{N}$-Jason, a run-time norm compliant BDI agent framework supporting norm-aware deliberation as well as run-time norm execution mechanism, through which new unknown norms are recognised and bring about the triggering of plans. To be able to process a norm such as an obligation, the agent architecture must be able to deal with deadlines and priorities, and choose between plans triggered by a particular norm. Consequently, we extend the syntax and the scheduling algorithm of AgentSpeak(RT) to operate in the context of Jason/AgentSpeak(L) and provide 'real-time agency', which we explain through a detailed examination of the operational semantics of a single reasoning cycle.
\end{abstract}

Keywords: Norms, BDI, Agent Programming Language, Normative System

\section{Introduction}

In conventional BDI agents, norm compliance is typically achieved by design. That is by specifying plans that are triggered by detached norms, because the agent programmer knows which norms the agent will adopt, and then prioritising those rules so that those supporting norms are chosen over those preferred by the agent's mental attitudes, in order to suppress conflicts between the normative and the agent's existing goals. This creates an undesirable dependence between the agent implementation and the norm implementation, which creates two issues:

1. When an agent encounters new and unknown norms, which were not taken into account at design time, there is typically no plan to deal with those norms in the 
plan library at run-time. Hence, norm compliant behaviour cannot normally be exhibited because the norms are unavoidably ignored. Yet worse, agents may suffer a punishment from the enforcement of the normative system as a result of a violation caused by their incapacity to process the normative event.

2. The hierarchical prioritisation of normative over ordinary plans deprives an agent of its autonomy, since the norms in effect are treated as hard constraints, whose violation is not possible.

We believe that such tensions can be resolved by the use of an extended model of norm awareness. In the literature on BDI agents, norm awareness, which is a precursor to norm compliance, is exhibited broadly by two approaches: (i) at the perception level, by taking new unknown norms into account as part of the generic execution mechanism $[13,14]$ and (ii) at the deliberation level, by attempts to resolve the conflict between normative factors and agents' mental attitudes [1,9]. We propose to coalesce these approaches into one 'sense-think-act' reasoning cycle informed by the concept of awareness, which Charlton [4] describes as the capacity "to select and integrate relevant inputs from a complex environment to enable humans or animals to choose between a large repertoire of behavioural responses". This definition reminds us that, in order to be norm aware, agents should have knowledge (or understanding) about norms in respect of: (i) what (state) the norms intend to reach or to achieve, (ii) which action plans are appropriate to execute norms and (iii) which behaviour agents should prefer between normative goals and the agent's own interests.

Thus, this paper addresses the convergence of these approaches in the context of the BDI agent architecture, in order to be able to ground the discussion of how the extended model of norm awareness enables a BDI agent to exhibit norm compliant behaviour at run-time. To do so, we propose $\mathrm{N}$-Jason, a run-time norm-compliant BDI agent framework supporting a run-time norm execution mechanism, under which new and unknown norms are recognised and enable the triggering of an appropriate plan (if present), in conjunction with norm-aware deliberation [1]. To be able to process a norm such as an obligation, the agent architecture should be able to deal with deadlines and priorities, and choose between plans triggered by a particular norm. Consequently, we extend the syntax and the scheduling algorithm of $\operatorname{AgentSpeak(RT)~[15]~to~operate~in~the~context~}$ of Jason/AgentSpeak(L) [3] and provide 'real-time agency', which we explain through a detailed examination of the operational semantics of a single reasoning cycle.

The paper is organised as follows. In $\$ 2$ an institutional framework and semantics of norms considered in $\mathbf{N}$-Jason are introduced. It is followed by $\S 3$, where we present a run-time norm compliant BDI agent framework including programming language and interpreter. After the operational semantics in $\S 4$, related work and the contribution of this work are contrasted in $\S 5$. The conclusion and future work are discussed in $§ 6$.

\section{Institutional Framework}

Normative frameworks can be viewed as a kind of external repositories of (normative) knowledge from which (normative) guidance may be delivered to agents. Usually, a normative framework is composed of a set of rules whose purpose is to model the normative 
positions established by the actions of agents and hence realise the governance of individual agents in the society. These rules are not hard-coded recipes presenting reactive behaviours, such as those in the static expert systems, but rather describe consequences arising from observations for the purpose of reasoning about the current context, resulting in situation-specific norms. The framework identifies not only correct and incorrect actions but also norms such as obligations, permissions and prohibitions through the institutional trace that records its evolving internal state, subject to observed external events representing actions in the external world.

Depending on the formalism of the normative system, norms can be categorised as state- or event-based. State-based norms usually express higher level norms that impose desirable or required states on the system (or an environment), often as a logical combination of institutional facts, which should be brought about by the actions of agents [8]. In contrast, event-based norms generally represent relatively lower level activities addressing possibly executable events (or actions) at the individual agent level [7]. In this paper, we use Cliffe's institutional model [5] for the purpose of providing detached event-based norms, upon which we develop the run-time norm compliance model presented here.

The institutional framework provides a formal action language Inst $A L$ to specify norms, describing coordinations and interactions between agents and (or) environments in the context of an institution. The normative specification is translated to a computational model that utilises Answer Set Programming (ASP) [10], which enables reasoning about the current context described in the institution. The institution is composed of a set of institutional states, evolving over time triggered by the occurrence of both internal and external events. An institutional state is a set of fluents which are present (denoting true) or absent (denoting false) at a given time instant. In addition, such institutional fluents are divided into domain fluents and normative fluents which latter is made up of: (i) power $(\mathcal{W})$ - indicates events that are empowered to bring about institutional change (ii) permission $(\mathcal{P})$ - indicates events that can occur without violation, and (iii) obligations $(\mathcal{O})$ - specifies events that are obliged to happen before the occurrence of a deadline (e.g. a timeout), or else a violation occurs.

These normative fluents represent the normative consequences of particular behaviours which should be achieved by agents in a certain context. For example, if an agent $X$ is obliged to carry out an action act by deadline deadline otherwise the violation event violation is generated, the form of the normative information is represented as:

$$
\text { obl(act, deadline, violation) (obligation) }
$$

Also if an agent $X$ is permitted to perform an action $a c t$, then the representation is:

perm(act)

(permission)

The determination of those normative consequences is carried out using an answer set solver driven by a rule-based specification (Inst $A L$ ) which explores all possible outcomes derivable from the institutional state arising from the occurrence of a single 
event ${ }^{3}$ as determined by the generation and consequence rules that comprise the institutional model. Lee et al. [12] demonstrate a governance mechanism using this institutional model that shows how the normative consequences of particular actions can be delivered to agents' minds as percepts (to conventional Jason agents rather than the variety described here) either on request or by subscription, making them available for the agent reasoning process.

Van Riemsdijk et al. suggest in [14], that one feasible approach for run-time norm execution is the use of "pre-existing capabilities" in the agent program when an agent encounters new and unknown norms. This assumes that event-based norms can identify the associated necessary actions, since event-based norms typically refer to relatively low-level activities that address possibly executable events (or actions) at the individual agent level [7]. If appropriate information can be extracted from the detached norm, such that it is recognisable to an agent, an agent presumably may execute unknown norms and so exhibit norm compliance at run-time. For example, the act term in an obligation represents a similar level of knowledge to plans or events in a BDI agent program. If an agent can retrieve and recognise what action (or event) is required to be achieved, then it can trigger certain plans and attempt to carry out such behaviour even though the norm is not handled explicitly in the agent specification. With regard to the norm-aware reasoning, an agent may deduce a preference, if it is able to know the relative priorities, and critical impact or the deadline of normative factors by extracting deadline and violation information. This norm-aware reasoning may allow an agent to pursue its own preferences between its own goals, norms and sanctions by measuring feasibility, as proposed by Alechina et al. [1]. In this paper, we only use obligations for such purpose, in order to focus on the essential aspects of the agent's internal reasoning process. Additionally, we consider the handling of prohibitions for the compatibility with other normative systems, however they are not explicit in the institution mechanism employed here.

\section{The N-Jason BDI Agent Framework}

In this section we outline $\mathrm{N}$-Jason, a norm aware BDI agent interpreter and its programming language for run-time norm compliant agent behaviour. In principle, it extends Jason/AgentSpeak(L) syntactically, semantically and in the reasoning process of the interpreter. In practice, $\boldsymbol{N}$-Jason is conceptually similar to AgentSpeak(RT) [15], which is capable of dealing with deadlines and priorities and scheduling intentions with the aim of providing real-time agency. $N$-Jason is conceptually a superset of AgentSpeak(RT), to which it adds normative concepts (i.e. obligations, permissions, prohibitions, deadlines, priorities and durations) and norm aware deliberation.

We firstly examine work to date with regards to the programming language aspect. This is followed by an informal explanation of the $\mathrm{N}$-Jason reasoning cycle. Subsequently, we show how the extended model of norm awareness in BDI agents is estab-

\footnotetext{
${ }^{3}$ Note: the institutional model can also function as a normative oracle for an agent, if presented with a sequence of events, in which case it derives all the possible outcomes from all possible orderings of those events, subject to whatever constraints are specified on the ordering.
} 
lished by the combination of the run-time norm execution mechanism and norm-aware deliberation.

\subsection{The $N$-Jason Agent Programming Language}

A $N$-Jason agent consists of four main components: beliefs, goals, events and a set of plans. Beliefs and goals are identical to those in standard Jason, while events and plans are extended. We now give a brief summary of the extended features of the basic elements in the agent specification.

Belief: A belief represents knowledge about the environments wherein agents are situated. It is composed of percepts observed by agents, messages containing the information about other agents, and norms delivered from normative frameworks. Typically, a belief is represented as a grounded atomic formula. The collection of beliefs is referred to as a belief base, which contains belief literals decomposed into belief atoms and negations.

Goal: A goal is one of two basic types: an achievement goal and a test goal. The former are usually specified as predicates prefixed by the '!' operator. This specifies a certain state of the environment that the agent wants to achieve, which is indicated when the predicate associated with its achievement goal is true. The latter test goal, for which the prefix is the '?' operator, indicates that agents want to know whether the associated predicate is a true belief.

Event: An event is the main component for triggering agent's plans. In principle, changes in agent's mental attitudes (i.e. beliefs, goals and intentions) give rise to events. There are two types of events: one is an addition event denoted by ' + ', which means the addition of a belief or an achievement goal. The other is a deletion event denoted by '-', referring to a recantation of a base belief.

As in Jason, an addition event is categorised by a belief addition event denoted by ' + ' and a goal addition event jointly denoted by ' + ' and '!'. All external belief changes bring about belief addition events, so as to initiate the execution of corresponding plans. In contrast, the goal addition event results from both internal and external changes in goals. In other words, explicit goals from the users or other agents result in a goal addition event, but also a goal addition event an be generated by internal operations affecting the agent's mental attitude, such as the execution of subgoals triggered in response to an external event.

Support for normative concepts is provided by an extension of the syntax for an event by the addition of deadline and priority information. The deadline is a real time value indicating a deadline by which an intention should be achieved. It is expressed in a some adequate unit of real world time. When the deadline is passed, it is no longer feasible to achieve an intention or to give a response with a belief change. The priority is a positive integer value that expresses the relative importance between the achievement of an intention and responding to changes in a belief. A larger value reflects a higher priority. Both can be specified optionally in the annotation (a list of terms in between square brackets "[" and "]") at the end of an event. For example the event:

+ !at $(X, Y)[$ deadline $(900)$, priority $(10)]$ 
specifies the goal adoption that an agent moves to the coordinate $(\mathrm{X}, \mathrm{Y})$, by the deadline 900 , with priority 10 . The deadline is taken as infinity and the priority as zero, by default. Note that those annotated deadline and priority are not involved in an unification at plan selection stage.

Plan: A plan is a sequence of actions (and subgoals) which is a means to achieve a (main) goal or a means to respond with changes in beliefs by agents. The plan typically consists of a head and a body, but sometimes an optional plan label, which defines an index, a name and other information, can be specified. The head is composed of a triggering event, which specifies an event for which the plan is to be used and a context specifying the condition which must be true for the plan to be a candidate for execution. The body is a series of actions and subgoals to achieve a main goal.

The plan is extended to support normative concepts. Given the three main elements, a duration is proposed in $\mathbf{N}$-Jason, specifically in order to enable assessment of the feasibility of the plan associated with the deadline (see §3.4). The duration is a non-negative integer value representing a required time to execute the plan. In principle, the duration may be determined by the summation of an execution time of each external action in the plan body. For simplicity, we follow the assumption described in [1], that the estimated time for each external action is fixed and already known. Like deadline and priority, a duration can be optionally specified in the plan label in the form of an annotation (a list of terms in between square brackets "[" and "]"). For example, the plan:

$$
\begin{aligned}
& \text { aplan [duration (50)] } \\
& + \text { !at }(X, Y): \operatorname{req}(\mathrm{ag}) \\
& <-\operatorname{move} \text { toward }(X, Y) ; \operatorname{lack}(\mathrm{ag}) \text {. }
\end{aligned}
$$

is triggered by the request from the agent $a g$ to move to the coordinate $(\mathrm{X}, \mathrm{Y})$, and then to send back an acknowledgement to $a g$. The required (or estimated) execution time of the plan is 50 .

\subsection{The $N$-Jason Interpreter}

The interpreter plays an important role in the operationalisation of agent programs. The agent's belief base, intentions and events are manipulated by the interpreter, and practical reasoning consisting of deliberation and means-ends reasoning is performed to achieve a goal or to respond to environmental changes.

During a single reasoning cycle, run-time norm compliance is accomplished by an extended model of norm awareness that has three steps:

1. Event Reconsideration, to find out what the norm is intended to achieve or to reach,

2. Option Reconsideration, to identify which plan is the most appropriate in response to the norm and

3. Intention Scheduling to confirm the decision about which behaviour agent would prefer between goals, norms and sanctions.

We now give an informal explanation of one reasoning cycle in the interpreter (see Algorithm 1). At the beginning (lines 1-4), an agent perceives some knowledge about 


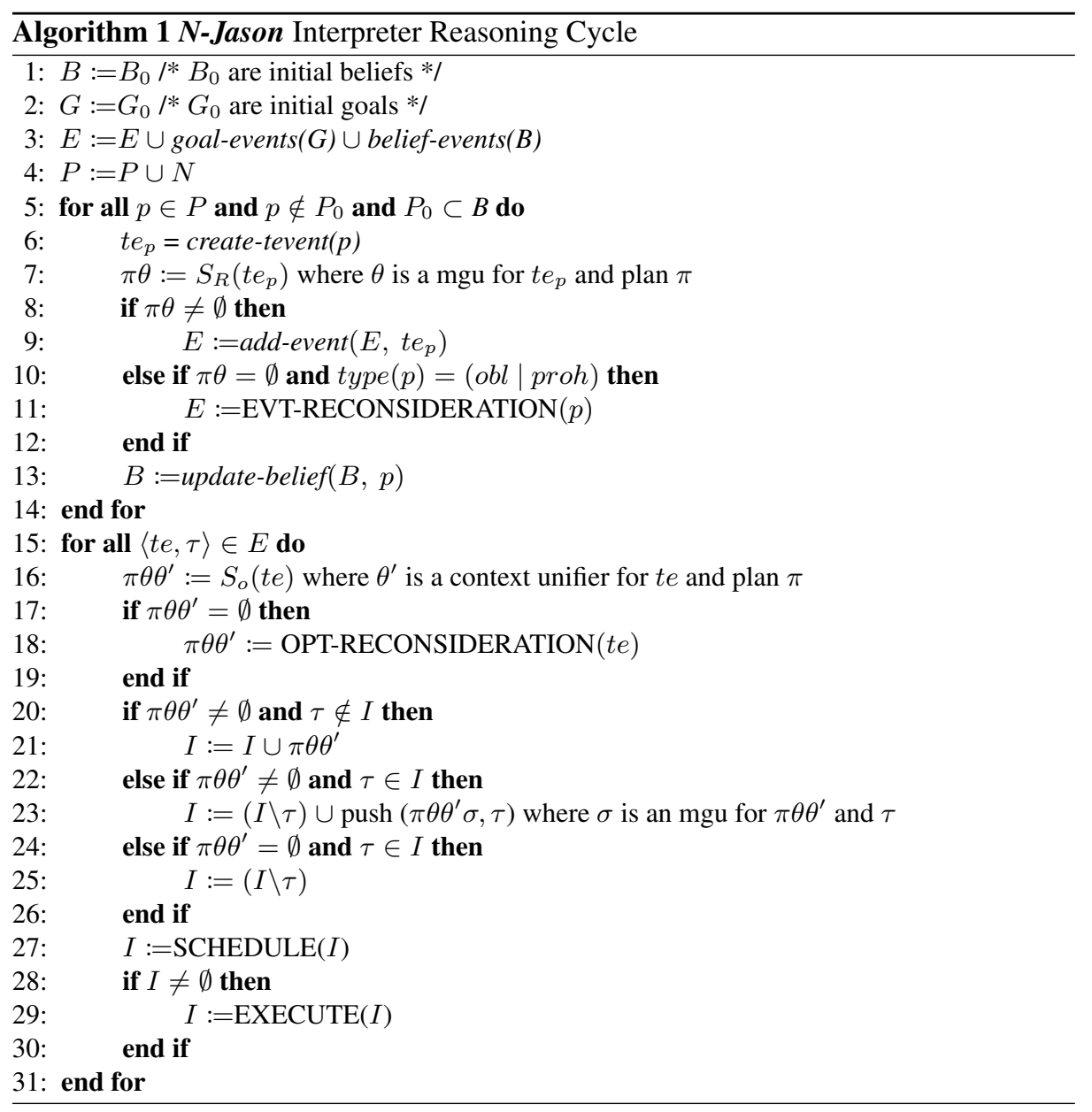

an environment $(P)$ as well as a list of norms $(N)$ (e.g. obligations) delivered from one or more institutional frameworks. Although those are separated entities at the agent level, it is unified into a set of percepts at the interpreter level (line 4). Thus, the interpreter may not be able to distinguish norms from percepts at this stage.

The belief base $(B)$ is updated by $P$ in the belief update process (belief-updatefunction (buf) more precisely) (see lines 6-13). This belief update involves the creation of events in response to each new percept. Once a percept $(p)$ is encoded as a triggering event $\left(t e_{p}\right)$, the interpreter checks whether $t e_{p}$ has relevant plans in the plan library $\Pi$ using the plan selection function $S_{R}$ (for more details about $S_{R}$, see [3]). If relevant plans are retrieved, then the event base $(E)$ is updated with $t e_{p}$.If no relevant plan is retrieved, $t e_{p}$ is ignored but $B$ is updated in any case with $p$. The same approach is taken for norms when the norms and its relevant plans are already specified in the agent program. Otherwise, the event reconsideration process (line 11) starts to find out what the norms are intended to achieve, as the first step in run-time norm execution. 
Next, the interpreter starts the reasoning process in order to determine an applicable plan in response to the te selected by the event selection function $S_{E}$. The selection function $S_{O}$ chooses a single option from the applicable plans as a result of the unification of event and context. If $S_{O}$ retrieves nothing, then the interpreter follows exactly the same path as described above. The option reconsideration process (line 18) tries to find out which action plans are appropriate to execute unknown norms, as the second step in run-time norm execution. See lines 16-19.

If one single applicable plan is successfully retrieved by $S_{O}$, then the means-ends reasoning adds the applicable plan $(\pi)$ as an intended means $(I M)$ on top of an intention (I). If te of $\pi$ is an internal event then $\pi$ added in the existing $I$, otherwise a new $I$ is created with $\pi$ to be added in there (line 20-26). This is followed by the intention scheduling process which returns a preference maximal set of intentions in deadline order (line 27). Afterwards, one intention selected by the intention selection function $S_{I}$ is finally executed (line 29). The details of the remainder are exactly the same as in [3] or [15].

The internal operation of the $\mathbf{N}$-Jason interpreter is shown in Algorithm 1 extended from [15]. We use the same notations as in [15] for consistency and comparability. The functions EVT- and OPT-RECONSIDERATION accomplish the run-time norm execution mechanism described in $\S 3.3$. The function $e d p$ constructs an event using the terms in the event-based norm, if the type of a percept $p$ is a norm (e.g. obligations). The main algorithm of the SCHEDULE function is shown in $\$ 3.4$.

\subsection{Run-Time Norm Execution}

In $\S 3.2$, we explained that run-time norm execution is realised by two steps: (i) event reconsideration and (ii) option reconsideration. Prior to defining those reconsideration processes, we firstly define a property of the executability of norms at run-time. We say that a norm such as obl (evt, deadline, violation), is executable at run-time iff:

1. $p \in P$ and $\operatorname{type}(p)=$ (obligation $\mid$ prohibition $)$, where $p$ is a percept, formed from a list of terms such as term("," term)*, in a set of newly observed percepts $P$ at run-time;

2. $t e_{p} \notin E$, where $t e_{p}$ is a triggering event generated from the percept $p$, and $E$ is an event base, which is a set of events $\left\{(t e, \tau),\left(t e^{\prime}, \tau^{\prime}\right), \ldots\right\}$, where an event is a pair of a triggering event and an intention $(t e, \tau)$;

3. $e d p(p) \neq \emptyset$ and $\left\{t e_{e d p(p)}\right\} \cap E \neq \phi$, where $e d p(p)$ is a function extracting the obliged event together with its deadline and priority from $p$ and $t e_{e d p(p)}$ is a triggering event of the $e d p(p)$, an event term in the norm, and

4. $R_{t e_{e d p(p)}} \neq \phi$, where $R_{t e_{e d p(p)}}$ is a relevant plans selection function.

The executability determines the necessity of further reconsideration for the new and unknown norms. If those norms are judged executable at the perception stage, the event-reconsideration process starts for the addition of such norms to the event base as triggering events. Similarly, the executability also enables the option-reconsideration in order to execute an applicable plan in relation to the triggering events derived from the norms. 


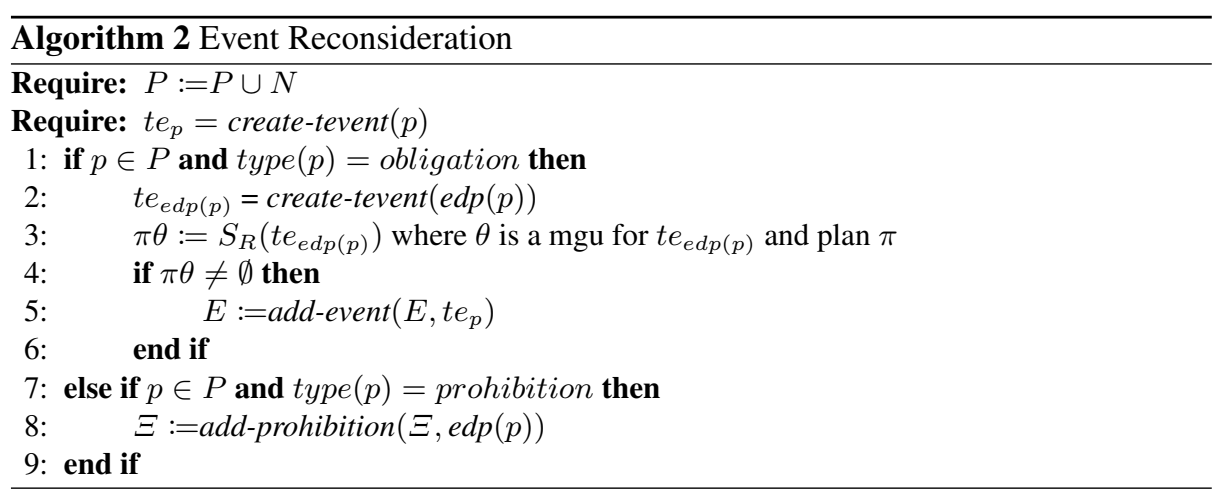

Event Reconsideration aims to verify that a norm perceived at run-time is executable although no corresponding plan exists in the agent program. If an event extracted from a detached norm has a relevance to a certain set of plans, it thus has potential to trigger specific ones, and it is then concluded that the norm is executable. If the norm is proven to be executable, the interpreter adds the norm to the event base $E$ as an achievement goal addition event. The procedure for event reconsideration is as follows (see Algorithm 2):

1. Extract the terms representing an obliged event, a deadline and its priority ${ }^{4}$ from the obligation by the function $e d p$, whose practical implementation may vary, depending on norm representations in various systems (line 2),

2. Construct a new triggering event (an achievement goal addition event in this case) from the combination of extracted terms (line 2),

3. Query the existence of a set of relevant plans to $S_{R}$ with such a constructed triggering event (line 3),

4. Add such triggering event to $E$, if relevant plans are successfully retrieved (line 5) and

5. If the norm is a prohibition, then the extracted event is added into the prohibition base $(\Xi)$ (line $7-8)$ and will be revisited at the norm deliberation stage ${ }^{5}$.

For example, suppose there is a detached obligation obl ( at (X, Y), 1030, 10). If relevant plans are not found in the agent program (plan library of an agent, to be precise) in response to the obligation, the function $e d p$ firstly extracts the event (at ( $X$, Y)), deadline (1030) and priority (10) from the obligation. Next, the interpreter constructs a new triggering event (an achievement goal addition event as described above) such as + ! at $(X, Y)$ [deadline (1030), priority $(10)]$ using the extracted information. Subsequently, the interpreter queries the existence of relevant plans to $S_{R}$ once again with a new triggering event, + ! at $(X, Y)$ [deadline (1030), priority (10) ].

\footnotetext{
${ }^{4}$ In principle, the last term is an event which arises when a violation occurs. This value normally indicates the criticality of such a violation. Higher values represents a higher priority.

${ }^{5} \mathrm{~N}$-Jason supports prohibitions as described above, and is therefore compatible with normative systems supporting prohibitions, but we note that the institutional model described in $\$ 2$ does not have an explicit representation of prohibition, but only the absence of permission.
} 
If the retrieval of relevant plans is successful, then the original event, + ! obl (at ( $X$, Y) , 1030, 10), is added to $E$.

One exceptional aspect in event-reconsideration is the addition of a deontic event $t e_{p}$ (which is a detached norm) instead of a normal event $t e_{e d p(p)}$ (which is a newly constructed triggering event) into the event base $E$. In so doing, we intend to distinguish norm-triggered intentions from ordinary intentions that normal events trigger, so as to facilitate norm-aware deliberation (see §3.4) in $\mathbf{N}$-Jason. In principle, Jason creates different intentions in response to different triggering events. Given this characteristic, both a deontic and a normal event create a deontic and a normal intention in $\mathbf{N}$-Jason, respectively. The intended means included in both intentions are identical since a deontic and a normal event trigger exactly the same plan in an agent program. However, the properties (e.g. deadline and priority) of each intention are different. The normal intention follows the original deadline and priority specified in the plan. In contrast, the deontic intention has different deadline and priority, which are inherited from those in the detached norm. As a result, these intentions are the main source of norm-aware deliberation. An agent is able to deliberate on norms and agent's private goals through the evaluation of the relative importance and urgency using norm-triggered (i.e. deontic) intentions and ordinary event-triggered (i.e. normal) intentions.

Suppose a plan whose label is example, is specified in an agent program:

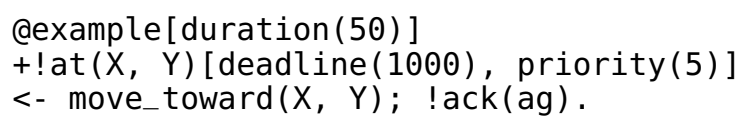

Assuming that a normal event triggering example is added to event base $E$. Then it creates a normal intention using a pair of normal event and its associated plan plan_example, whose deadline and priority are 1000 and 5, respectively. Later, a detached obligation obl (at $(X, Y), 1030,10$ ) is received. Following Algorithm 2, the deontic event + ! obl ( $\operatorname{at}(X, Y), 1030,10$ ) is added to $E$, since a relevant plan example is found. Consequently a deontic intention is created using a pair of a deontic event and its associated plan example. Its deadline and priority are 1030 and 10, respectively, which are different from those in the normal intention. Obviously, we have two intentions whose properties are different, although the intended means are absolutely same. Hence, $N$-Jason is able to carry out norm-aware deliberation on norms and the agent's own goals using those intentions. If $\mathbf{N}$-Jason simply adds a normal event instead of a deontic event when an obligation is detached, then norm-aware deliberation may not be feasible since there must be only one normal intention.

Option-Reconsideration is a central element in the practical reasoning process whereas the event reconsideration happens at the perception stage. The main objective of option reconsideration is the determination of an applicable plan corresponding to the new and unknown norm - whose executability is already verified - and is thus added to $E$ as an achievement goal addition event. If the applicable plan is chosen, then it will probably be used to enact a norm-compliant behaviour, unless it is infeasible as judged by intention scheduling (described in §3.4). The procedure is shown in Algorithm 3.

Like Event-Reconsideration, $t e_{p}$ is generated by a new and unknown norm that does not have any relevant plans $R_{t e_{p}}$ at this moment. Thus at the beginning of the option reconsideration, the interpreter carries out the same process for event reconsideration: 


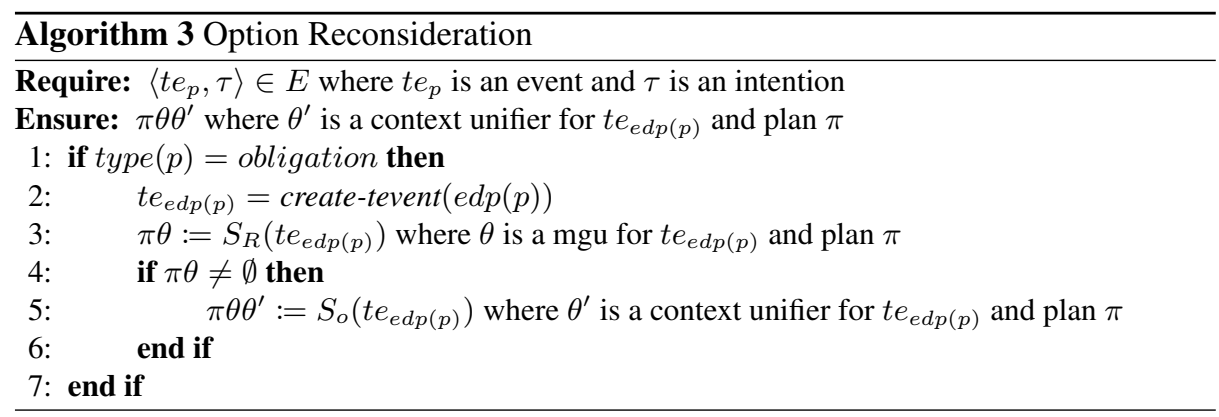

1. Extract the event term $e d p(p)$ of the norm in order to retrieve relevant plans $R_{t e_{e d p(p)}}$ (as before), if the type of $p$ is a norm (i.e. an obligation) (line 1 - 2),

2. Retrieve the relevant plans corresponding to the $R_{t e_{e d p(p)}}$ by the unification of an atomic-formula in a triggering event and each plan in an agent (line 3),

3. Determine a set of applicable plans through the extended unification of a triggering event, a plan and a context and (line 5) and

4. Select a single applicable plan as an intended means to which to commit (line 5).

\subsection{Norm Awareness in Deliberation}

Norm awareness in the deliberation process is achieved by the scheduling of intentions with deadlines and priorities. We extend the algorithm proposed in [15] with the consideration of prohibitions in order to establish a conflict-free preference maximal set of intentions. In effect, this is like [1] who proposes a scheduling algorithm that brings about a preference maximal set of intentions, but that depends upon (N-)2APL's parallel execution of plans, whereas here the scheduling algorithm for $(\mathrm{N}-)$ Jason has to take account of the single-threaded plan execution model in Jason.

The scheduling algorithm is introduced in Algorithm 4. A set of candidate intentions $I_{C}=\left\{\tau, \tau^{\prime}, \ldots\right\}$, which is sorted in descending order of a priority, is inserted into a scheduling process. If each intention is feasible, i.e. a plan on top of the intention can be executed before the deadline and is not prohibited by a set of prohibition $\Xi=$ $\left\{\xi, \xi^{\prime}, \ldots\right\}$, then the intention is added to the preference maximal set $(\Gamma)$ whose criteria are defined as follows:

1. An intention is feasible iff the execution of the intention is completed before its deadline, that is, for $\tau$,

$$
n e(\tau)+e t(\tau)-e x(\tau) \leq d l(\tau)
$$

where $\tau$ denotes an intention, $n e(\tau)$ is the time at which $\tau$ will next execute, $\operatorname{et}(\tau)$ is the time required to execute $\tau$, denoted in the plan label, $\operatorname{ex}(\tau)$ is the elapsed time to execute $\tau$ to this point, and $d l(\tau)$ is the deadline for $\tau$ specified in the plan [1].

2. The intention should not be prohibited, that is, for $\tau$

$$
\text { - } \tau \notin \Xi \text { or }
$$




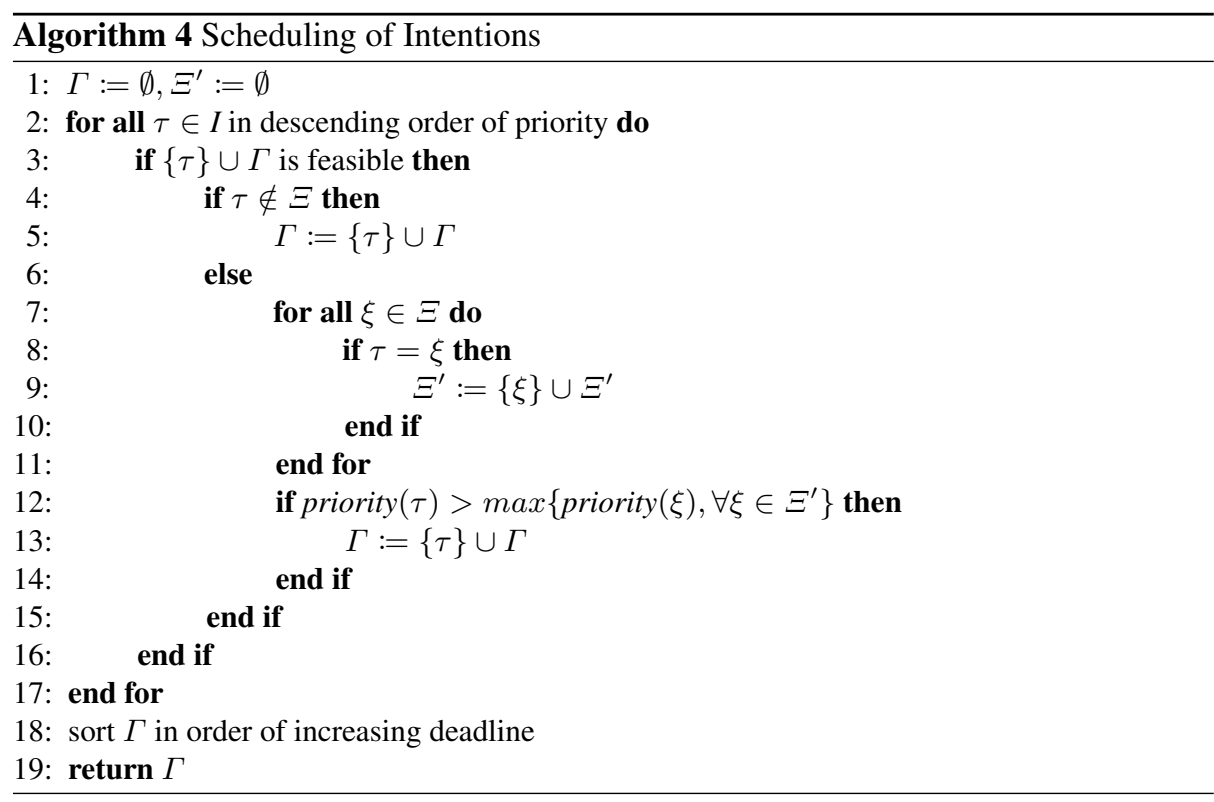

- $\tau \in \Xi$, then $\forall \xi \in \Xi, \tau=\xi$ and priority $(\tau)>\max \{\operatorname{priority}(\xi), \forall \xi \in \Xi\}$

where $\tau$ is an intention, $\xi$ is a prohibited event in the prohibition base $\Xi$ and priority is a priority retrieval function.

Scheduling in $\mathbf{N}$-Jason is also pre-emptive in that the adoption of a new intention $\tau$ may prevent scheduled intentions with lower priority than $\tau$ (including currently executing intentions) being added to the new schedule just as in N-2APL and AgentSpeak(RT). Intentions that cannot meet their deadline are dropped.

\subsection{Example}

As an example, we consider robots serving beer in a pub, whose main role is to get an order and to deliver a beer to the customer. We assume the existence of some institutions delivering desirable social norms, subject to the observations of participants, and that all agents are governed by such systems. A part of the agent program is shown below:

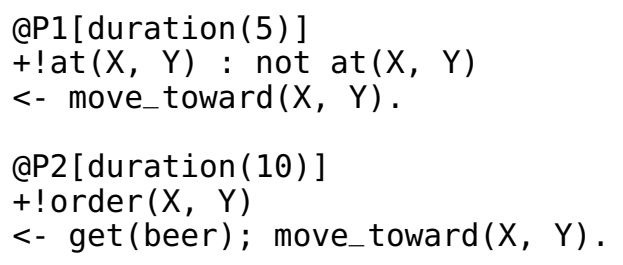

At time 100, the robot receives the following events:

E1: + ! request $(2,3)$ [deadline (130), priority (20)] A request from customer seated at $(2,3)$. The deadline is 130 and the customer is important so the priority is 20 . 
E2: + ! request $(1,1)$ [deadline (115), priority (10)] A request from customer seated at $(1,1)$. The deadline is 120 and the the priority is 10 .

E3: + ! request $(3,3)$ [deadline (130), priority (10)] A request from customer seated at $(3,3)$. The deadline is 130 and the the priority is 10 .

These three events trigger the plan $\mathrm{P} 2$, and give rise to three possible intentions $\tau_{1}$ ( $\mathrm{P} 2$ triggered by $(2,3)), \tau_{2}(\mathrm{P} 2$ triggered by $(1,1))$ and $\tau_{3}(\mathrm{P} 2$ triggered by $(3,3)) . \tau_{2}$ is not feasible, thus it is dropped, whereas $\tau_{1}$ and $\tau_{3}$ are feasible, so scheduled in deadline order: $\tau_{1}$ is scheduled first between 100 and 110 since it has an earlier deadline followed by $\tau_{3}$ between 110 and 120 . Now the agent starts the execution of $\tau_{1}$.

Let consider an announcement of a fire alarm by one of the normative frameworks. It broadcasts an obligation containing the coordinates of an exit to all participants so they may escape from the building. Suppose the norm is obl $(\operatorname{at}(0,0), 115,100)$. Although the obligation is not stated in the agent's program, it is executable since the agent has a pre-existing moving ability ! at $(X, Y)$, which is enough to satisfy the obligation. With the event- and option-reconsideration, the event :

E4: + ! at $(0,0)$ [deadline $(115)$, priority $(100)]$ is generated from the obligation, thus adoption the plan $\mathrm{P} 1$, bringing about an intention $\tau_{4}$ (P1 triggered by $(0,0))$. During the execution of $\tau_{1}, \tau_{3}$ and $\tau_{4}$ are inserted into a new schedule in deadline order: since the priority of $\tau_{4}$ is greater than $\tau_{3}$ and $\tau_{4}$ has a more urgent deadline, the agent starts to execute $\tau_{4}$, triggered by the obligation, before the execution of $\tau_{3}$.

Notwithstanding, that this example is extremely simple, it provides a useful inprinciple illustration of norm-aware deliberation - as performed by intention scheduling - as well as the run-time norm execution mechanism in $\mathbf{N}$-Jason.

\section{Operational Semantics}

In this section, we present a theoretical foundation for the $N$-Jason programming language with semantics based upon an extension of the operational semantics for $\mathbf{J a}$ son/AgentSpeak(L). Given the formal semantics of Jason we extend the transition rules which transform one extended configuration into another. To begin with, we show a configuration of individual $\mathbf{N}$-Jason agents which is almost unchanged except for norm configuration. In the following section, we describe the transition rules that give rise to a configuration change at each state in a single reasoning cycle. For consistency and comparability, we follow exactly the same notations as those in published Jason descriptions excepting the normative aspects.

\subsection{N-Jason Configuration}

The configuration of $\boldsymbol{N}$-Jason is a tuple $\langle a g, C, N, T, s\rangle$ where:

- $a g$ is an agent program consisting of a set of beliefs $b s$ and a set of plans $p s$, as defined by the EBNF in [3]. 
- An agent's circumstance $C$ is a tuple $\langle I, E, A\rangle$, where $I$ is a set of intention $\left\{i, i^{\prime}, \ldots\right\}$, $E$ is a set of events $\left\{(t e, i),\left(t e^{\prime}, i^{\prime}\right), \ldots\right\}$, in which event is a pair of a triggering event and an intention $(t e, i)$ and $A$ is a set of actions an agent performs in the external environment.

- $N$ is a tuple $\langle\Gamma, \Xi\rangle$ denoting normative consequences delivered from normative systems, where $\Gamma$ is a set of obligations $\left\{\gamma, \gamma^{\prime}, \ldots\right\}$ and $\Xi$ is a set of prohibition $\left\{\xi, \xi^{\prime}, \ldots\right\}$.

- $T$ is a tuple $\left\langle R, A_{p}, \iota, \varepsilon, \rho\right\rangle$ defining a trace of provisional information required for subsequent steps within a single reasoning cycle, where $R$ is the set of relevant plans, $A_{p}$ the sets of applicable plans, and $\iota, \varepsilon$ and $\rho$ record an intention, event, and applicable plan (respectively) at a specific moment under consideration within the execution of a single reasoning cycle.

- The current state $s$ within an agent's reasoning cycle is denoted by $s \in\{$ RcvNorm, SelEv, RelPI, AppIPI, AddIM, SchInt, Sellnt, Execlnt, ClrInt\}.

\subsection{Transition Rules}

The execution of the $\mathbf{N}$-Jason program leads the modification of the initial configuration of an agent via transition rules given below. For the sake of brevity, we do not repeat the communication semantics, since these are unaffected by the changes in relation to norms.

In general, the transition would normally start from the state ProcMsg, but we propose a preceding step RcvNorm, as described in $\$ 2$. Thus, note that the initial configuration of this model is $\langle a g, C, N, T, \operatorname{RcvNorm}\rangle$, where $a g$ is specified by the agent program and other all components are empty, and the reasoning cycle starts from RcvNorm with the transition rules given below.

Receiving detached norms: As described in $\S 2$, institutional frameworks may distribute norms via broadcasting when a norm is activated by the fulfilment of institutional states triggered by external events in the environment. As soon as the event-based norms are received, the norms effectively act like an ordinary event thus trigger the transition of the agent's mental state. Rule 4.2 updates the agent belief base and an event base component $C_{E}$ associated with adding new norms, specifically in case of obligations in an obligation base $N_{\Gamma}$. Otherwise, only a prohibition is added into the prohibition base and there are no updates to other components.

$$
\begin{gathered}
\frac{N \neq\{\} C_{E} \neq\{\}}{\langle a g, C, N, T, \text { RcvNorm }\rangle \rightarrow\left\langle a g^{\prime}, C^{\prime}, N^{\prime}, T, \text { SelEv }\right\rangle} \\
\text { where: } \quad a g_{b s}^{\prime}=a g_{b s} \cup\{\gamma\} \\
N_{\Gamma}^{\prime}=N_{\Gamma} \cup\{\gamma\} \vee N_{\Xi}^{\prime}=N_{\Xi} \cup\{\xi\} \\
C_{E}^{\prime}=C_{E} \cup\{\langle\gamma, i\rangle\}
\end{gathered}
$$

(RevNorm)

Relevant plans: If the transition of states (RcvNorm $\mapsto$ SelEv) is successful after RcvNorm and the state SelEv selects one event from the component $E$ of which event is either $\langle t e, i\rangle$ or $\langle\gamma, i\rangle$, rule 4.2 starts to assign the set of relevant plans to component $T_{R}$ in the state RelPI. Rule 4.2 indicates the reconsideration situation where a new 
triggering event extracted from the obligation is assigned to the component $C_{E}$, where $\operatorname{Evt}(\gamma)$ is a function constructing a triggering event by the retrieval of information from $\gamma$. Rule 4.2 assigns a set of relevant plans to $T_{R}$ in respect of the reconsidered event. Rule 4.2 and 4.2 cope with the situation where no relevant plan is retrieved. In those cases, events (both ordinary event and reconsidered event) are simply ignored and the state returns to SelEv.

$$
\begin{aligned}
& \frac{T_{\varepsilon}=\langle t e, i\rangle \quad \text { RelPlans }\left(a g_{p s}, t e\right) \neq\{\}}{\langle a g, C, N, T, \text { RelPI }\rangle \rightarrow\left\langle a g, C, N, T^{\prime}, \text { ApplPI }\right\rangle} \\
& \text { where: } \quad T_{R}^{\prime}=\operatorname{RelPlans}\left(a g_{p s}, t e\right) \\
& \frac{T_{\varepsilon}=\langle\gamma, i\rangle \quad \operatorname{RelPlans}\left(a g_{p s}, \gamma\right)=\{\}}{\langle a g, C, N, T, \text { RelPI }\rangle \rightarrow\left\langle a g, C^{\prime}, N, T, \text { RelPI }\right\rangle} \\
& \text { where: } \quad C_{E}^{\prime}=\{\langle\operatorname{Evt}(\gamma), i\rangle\} \\
& \frac{T_{\varepsilon}=\langle\operatorname{Evt}(\gamma), i\rangle \operatorname{ReIPlans}\left(a g_{p s}, \operatorname{Evt}(\gamma)\right) \neq\{\}}{\langle a g, C, N, T, \operatorname{Re} I \mathrm{PI}\rangle \rightarrow\left\langle a g, C, N, T^{\prime}, \mathrm{ApplPI}\right\rangle} \\
& \text { where: } \quad T_{R}^{\prime}=\operatorname{RelPlans}\left(a g_{p s}, \operatorname{Evt}(\gamma)\right) \\
& \frac{\operatorname{RelPlans}\left(a g_{p s}, t e\right)=\{\}}{\langle a g, C, N, T, \operatorname{RelPI}\rangle \rightarrow\langle a g, C, N, T, \text { SelEv }\rangle} \\
& \frac{\operatorname{RelPlans}\left(a g_{p s}, \operatorname{Evt}(\gamma)\right)=\{\}}{\langle a g, C, N, T, \operatorname{ReIPI}\rangle \rightarrow\langle a g, C, N, T, \text { SelEv }\rangle}
\end{aligned}
$$

Since transition rules between (AppPI $\mapsto$ AddIM) are almost same as those in $\mathbf{J a}$ son we give a brief description of each rule at each state from here. If $T_{R}^{\prime}$ is successfully assigned then it is followed by: (i) AppPI which assigns a set of applicable plans to $T_{A_{P}}$ by retrieving those relevant plans whose contexts are believed to be true, (ii) SelAppl which assigns a particular intended means selected by an option selection function $S_{O}$ to $T_{\rho}$, and (iii) AddIM which adds a selected intended means to $C_{I}$ which is an existing intention or a newly created one. If transitions fail between (AppPI $\mapsto$ AddIM), then the state Sellnt becomes the next step. For more information, see [3].

Scheduling of intentions: Rule 4.2 updates the component $C_{I}^{\prime}$ by the function $\operatorname{SCHEDULE}\left(C_{I}\right)$. Note that the scheduling function, $\operatorname{SCHEDULE}\left(C_{I}\right)$, sorts intentions in order of priority and deadline so as to determine the preference maximal set of intentions discussed in $\S 3.4$.

$$
\begin{array}{r}
\frac{T_{\rho}=\{\}}{\langle a g, C, N, T, \text { Schlnt }\rangle \rightarrow\left\langle a g, C^{\prime}, N, T, \text { Sellnt }\right\rangle} \\
\text { where: } \quad C_{I}^{\prime}=\operatorname{SCHEDULE}\left(C_{I}\right)
\end{array}
$$

After this step, the transition system follows the same rules as presented in [3] in order to execute an intended means in an particular intention selected by $S_{I}$ in between Sellnt, Execlnt and ClrInt. 


\section{Related Works}

There has been much research over a number of years on the matter of norm compliance through the combination of normative frameworks and classical (BDI-type) cognitive agents $[2,11]$. However, research on compliance of norms at the individual agent level has received less attention. As discussed in $§ 1$, this problem can be decomposed into two perspectives: to facilitate a generic norm execution mechanism at run-time, and to focus on the rational decision making between norms and existing goals.

Alechina et al. [1] introduce N-2APL, a norm-aware BDI agent architecture and its programming language. It is able to carry out norm-aware deliberation, which aims to permit agents to resolve the conflicts between an agent's own goals, normative goals and sanctions. This is accomplished by a deadline- and priority-based intention scheduling algorithm, which weighs the feasibility for all intentions that may bring about conflicts. The (potential) sanctions may affect agent decision making, but violations are possible in this approach. Given N-2APL, Dybalova et al. [9] demonstrate norm-compliant agents in location-based gaming environments in conjunction with the organisational framework, 2OPL [6]. There, once organisations have broadcast state-based norms to all participants, the individual agents achieve a state of the environment described in the norms using a design-based approach. $\mathbf{N}$-Jason is also able to support norm-aware deliberation in conjunction with an institutional model, which is similar to the combination of N-2APL and 2OPL, but extends the concept of norm awareness to the whole reasoning cycle. As a result, it supports agents in being design-based norm compliant, but can additionally deliver run-time compliance through norm execution.

Meneguzzi et al. [13] focuses on norm awareness at the perception level, by extending the AgentSpeak(L) BDI architecture with a run-time plan modification technique. It enables agents to behave appropriately in response to newly accepted norms at runtime. However, it assumes that the norms are non-conflicting, so it does not consider scheduling of plans with regards to their deadlines or possible sanctions in accordance with existing goals in agents. Whereas [13] takes a rather practical perspective, van Riemsdijk et al. [14] introduce a formal framework for generic norm execution, which allows agents to be norm compliant by triggering or preventing actions in new and unknown norms at design time. However the agent in [14] works at the level of individual actions (its decision mechanism chooses actions rather than plans) and the norms are specified in terms of actions, making in effect a norm-reactive agent, and it is unclear how the decision mechanism can combine actions to achieve goals and thereby the objective of a norm-deliberative agent. In $N$-Jason, run-time norm execution is in practice accomplished at the level of plans to achieve goals, and norms indicate a sort of event that triggers plans. Moreover, in $\mathbf{N}$-Jason run-time norm compliance is achieved on top of the norm aware decision making and in conjunction with the execution mechanism.

Notwithstanding the benefits of $\mathbf{N}$-Jason, there are some issues to discuss, particularly regarding the mechanism for run-time norms. In the run-time norm execution, the norm compliance strategy is hard-coded in the semantics of the language. whereas such a strategy is programmable as agent plans (i.e. supporting the design of strategy by an agent programmer) in JaCaMo [2] and N-2APL [1]. Thus, the proposal in this paper somewhat simplifies normative reasoning, since it deprives agents of a flexibility to change the plans dynamically or mis-behave intentionally, based on rules the agent pro- 
grammer designs. However, the mechanism we propose can enable legacy agents which have no compliance rule or strategy in their specification to become norm-aware automatically. Thus, those agents' behaviour can be coordinated through the governance of normative frameworks without further engineering efforts.

Another issue lies in the simple mechanism for the operationalisation of norms in run-time norm execution. The approach described here means the ontology and syntax of norms that can be executed are limited to those present in the plan library of an agent. In consequence, some detached norms, that may correspond semantically to one of an agent's plans, but which are ontologically different from the plan, will be ignored or violated. We are considering how to generalise the execution mechanism with the analysis of semantics of norms, following [14], in conjunction with plan synthesis.

\section{Conclusion and Future Works}

In this paper, we have presented a design for a norm-aware BDI agent, $N$-Jason, that enables the exhibition of norm compliance at run-time. Basically $\mathbf{N}$-Jason offers a generic norm execution mechanism on top of norm-aware deliberation to contribute to the exploitation of run-time norm compliance. Run-time norm execution specifically focuses on the operationalisation of new and unknown (event-based) norms not stated in the agent program at run-time. By judging the executability of them, $N$-Jason agents executes those norms following an extended model of norm awareness consisting of: (i) event reconsideration, to find out what the norm is intended to achieve or to reach, and (ii) option reconsideration, to identify which plan is the most appropriate in response to the norm. The selection of norm compliant behaviour is achieved in the norm-aware deliberation process by intention scheduling with deadlines, priorities and prohibitions which confirms the decision about which behaviour agent would prefer between goals, norms and sanctions. It brings about a preference maximal set of intentions in order to realise the norm compliance. $\mathbf{N}$-Jason is implemented in $\mathbf{J a}$ son/AgentSpeak(L) and extends its syntax and semantics to create $\mathbf{N}$-Jason.

We believe that run-time norm compliance model is beneficial for the enhancement of both a norm compliance capability and agent autonomy from the agent's perspective. However, we note that the behaviour triggered by run-time norm execution may look like unpredictable/unwanted behaviour from the agent programmer's perspective.

Although this paper particularly considers the execution of event-based norms at run-time in conjunction with the institutional model, the extension to support statebased norms and its normative systems can easily be incorporated into $\mathrm{N}$-Jason agents and will be as future work. We also plan to detect violations which are generated in the norm aware deliberation, particularly when the normative goals are dropped during scheduling. This offers a potentially useful link for enforcement in the context of normative system implementation. In addition, both empirical and analytical evaluation of the performance of $\mathbf{N}$-Jason requires proper investigation.

\section{References}

1. N. Alechina, M. Dastani, and B. Logan. Programming norm-aware agents. In Proceedings of the 11th International Conference on Autonomous Agents and Multiagent Systems, pages 
1057-1064, Richland, SC, 2012.

2. O. Boissier, R. H. Bordini, J. F. Hübner, A. Ricci, and A. Santi. Multi-agent oriented programming with jacamo. Sci. Comput. Program., 78(6):747-761, June 2013.

3. R. Bordini, M. Wooldridge, and J. Hübner. Programming Multi-Agent Systems in AgentSpeak using Jason (Wiley Series in Agent Technology). John Wiley \& Sons, 2007.

4. B. Charlton. Evolution and the cognitive neuroscience of awareness, consciousness and language. Cognition, 50:7-15, 2000.

5. O. Cliffe, M. De Vos, and J. Padget. Specifying and reasoning about multiple institutions. In P. Noriega, J. Vázquez-Salceda, G. Boella, O. Boissier, V. Dignum, N. Fornara, and E. Matson, editors, Coordination, Organizations, Institutions, and Norms in Agent Systems II, volume 4386 of Lecture Notes in Computer Science, pages 67-85. Springer Berlin Heidelberg, 2007.

6. M. Dastani, N. A. Tinnemeier, and J.-J. C. Meyer. A programming language for normative multi-agent systems. Multi-Agent Systems: Semantics and Dynamics of Organizational Models, pages 397-417, 2009.

7. M. De Vos, T. Balke, and K. Satoh. Combining event-and state-based norms. In Proceedings of the 2013 international conference on Autonomous agents and multi-agent systems, AAMAS '13, pages 1157-1158, Richland, SC, 2013. International Foundation for Autonomous Agents and Multiagent Systems.

8. V. Dignum. A Model for Organizational Interaction. PhD thesis, Utrecht University, 2004.

9. D. Dybalova, B. Testerink, M. Dastani, and B. Logan. A framework for programming normaware multi-agent systems. In F. Dignum and A. Chopra, editors, Proceedings of the 15th International Workshop on Coordination, Organisations, Institutions and Norms (COIN 2013), 2013.

10. M. Gelfond and V. Lifschitz. Classical negation in logic programs and disjunctive databases. New Generation Comput., 9(3/4):365-386, 1991.

11. J. F. Hubner, J. S. Sichman, and O. Boissier. Developing organised multiagent systems using the moise+ model: programming issues at the system and agent levels. Int. J. Agent-Oriented Softw. Eng., 1(3/4):370-395, Dec. 2007.

12. J. Lee, T. Li, and J. Padget. Towards polite virtual agents using social reasoning techniques. Computer Animation and Virtual Worlds, 24(3-4):335-343, 2013.

13. F. Meneguzzi and M. Luck. Norm-based behaviour modification in bdi agents. In Proceedings of The 8th International Conference on Autonomous Agents and Multiagent Systems Volume 1, AAMAS '09, pages 177-184, Richland, SC, 2009. International Foundation for Autonomous Agents and Multiagent Systems.

14. M. B. van Riemsdijk, L. A. Dennis, M. Fisher, and K. V. Hindriks. Agent reasoning for norm compliance: a semantic approach. In Proceedings of the 2013 international conference on Autonomous agents and multi-agent systems, AAMAS '13, pages 499-506, Richland, SC, 2013. International Foundation for Autonomous Agents and Multiagent Systems.

15. K. Vikhorev, N. Alechina, and B. Logan. Agent programming with priorities and deadlines. In The 10th International Conference on Autonomous Agents and Multiagent Systems, pages 397-404, Richland, SC, 2011. 